\title{
Práticas para Tratamento de Fatores Críticos de Sucesso
}

\author{
Raphael Freire $^{1}$, Davi Viana ${ }^{2}$, Gleison Santos ${ }^{1}$ \\ ${ }^{1}$ Programa de Pós-Graduação em Informática - Universidade Federal do Estado do Rio \\ de Janeiro (UNIRIO) - Av. Pasteur, 458, Urca, CEP 22290-240 - Rio de Janeiro, RJ \\ ${ }^{2}$ Programa de Pós-Graduação em Ciência da Computação - Universidade Federal do \\ Maranhão (UFMA) - Av. dos Portugueses, 1966, Bacanga, CEP 65080-805 - São Luís, \\ MA \\ \{raphael.freire,gleison.santos\}@uniriotec.br, davi.viana@lsdi.ufma.br
}

\begin{abstract}
Organizations confront a series of difficulties conducting software process improvement (SPI) initiatives. The causes of such difficulties may be related to organizational, technological or sociocultural aspects. Some of these aspects are known as critical success factors. In this context, this research presents a catalog of practices that can address negative critical factors. The catalog was conceived from cycles of incremental learning, using the Design Science Research methodology. We performed two case studies in order to evaluate the catalog and we identified the relevance of $84 \%$ of the practices in SPI initiatives contexts.
\end{abstract}

Resumo. As organizações confrontam uma série de dificuldades na condução de iniciativas de melhoria de processos de software (SPI). As causas podem estar relacionadas a aspectos de cunho organizacional, tecnológico e sociocultural. Alguns desses aspectos são considerados fatores críticos de sucesso. Neste contexto, este trabalho apresenta um catálogo de práticas que podem ser utilizadas para tratar os fatores críticos negativos. Este catálogo foi concebido a partir de ciclos de aprendizado incrementais, utilizando a metodologia Design Science Research. Em uma avaliação do catálogo realizada por meio de dois estudos de caso, foi identificada a pertinência de $84 \%$ das práticas em relação aos contextos das iniciativas de MPS em questão.

\section{Introdução}

A aplicação das boas práticas de Engenharia de Software em iniciativas de Melhoria de Processo de Software (ou, SPI, do inglês software process improvement) tem se tornado uma estratégia constante em organizações de software para aumentar a qualidade dos seus produtos. As organizações partem da suposição de que a qualidade do produto pode ser elevada devido ao aumento da qualidade do processo de desenvolvimento (FUGGETTA, 2000; MONTONI e ROCHA, 2011). O sucesso na implementação de iniciativas de melhoria de processos de software depende fundamentalmente de estratégias e abordagens adotadas para apoiar a execução de tais iniciativas.

Entretanto, a falta de adequação dessas abordagens utilizadas é uma das razões para o fracasso das iniciativas de melhoria (MONTONI, 2010). Outros fatores sociais e culturais, como comunicação, falta de motivação e falta de apoio da alta direção também são apontados como causadores de fracassos na condução destas iniciativas 
(BAYONA et al., 2012). Além disso, aspectos relacionados com a equipe de consultoria, como falta de competências técnicas, falta de confiança no consultor e falta de abertura para ouvir opiniões, são fatores críticos que podem influenciar negativamente as iniciativas de melhoria de processos de software (MONTONI, 2010).

Diversos estudos foram realizados na área com o intuito de identificar as causas dos problemas que influenciam o sucesso de iniciativas de melhoria, bem como analisar suas interações, efeitos e ações de tratamento (DUTRA, 2015; BAYONA et al., 2013; BAYONA et al., 2012; MONTONI, 2010). Esses problemas são tratados, comumente, como Fatores Críticos de Sucesso (FCS), pois constituem um número reduzido de questões importantes em que a alta gerência deve colocar atenção para alcançar os resultados esperados com a implementação de melhorias nos processos (MONTONI, 2010). Contudo, poucos trabalhos buscam identificar práticas no sentido de mitigar os efeitos negativos dos FCS (MENDES et al., 2007).

A aplicação de práticas de Gerência de Conhecimento (GC) e Aprendizagem Organizacional (AO) e de práticas gerenciais pode ser uma estratégia utilizada para as organizações reagirem a estes fatores críticos de influência negativa. Práticas de GC e AO buscam organizar a identificação e utilização de conhecimento organizacional em benefício das atividades organizacionais (IANDOLI e ZOLLO, 2008; SENGE, 1991), enquanto as práticas gerenciais auxiliam nas questões organizacionais e culturais, como inserir o corpo funcional em atividades de conscientização para ressaltar a importância do projeto de melhoria (PARENTE et al., 2008).

Neste sentido, o objetivo deste trabalho é investigar quais práticas podem ser empregadas com o objetivo de reagir aos FCS causadores de fracassos em SPI. Neste trabalho, esses FCS são denominados de fatores críticos de influência negativa. Para realizar esta pesquisa foi adotada a metodologia Design Science Research (DSR), onde foram conduzidos ciclos de aprendizado incrementais com intuito de obter conhecimento em relação ao tratamento destes fatores. Como resultado, foram identificadas 135 práticas gerenciais, 27 práticas de $\mathrm{GC}$ e $\mathrm{AO}$ e 24 ferramentas que podem ser utilizadas neste contexto.

Além desta seção introdutória, o artigo está organizado da seguinte forma: Seção 2 apresenta uma revisão da literatura sobre FCS e conceitos sobre GC e AO; Seção 3 descreve a metodologia de pesquisa e os ciclos de aprendizado incrementais realizados; Seção 4 expõe o catálogo de práticas para tratamento dos fatores críticos negativos e discorre sobre as limitações do trabalho. Por fim, a Seção 5 apresenta as considerações finais.

\section{Fatores Críticos de Sucesso e Gerência do Conhecimento em Melhoria de Processos de Software}

A partir de uma revisão da literatura, foram identificados diversos estudos que apontam fatores críticos de sucesso de iniciativas de SPI (BAYONA et al., 2013; MONTONI, 2010; VIRTANEN et al., 2013; BAYONA et al., 2012; HABIB, 2009), dificuldades (ROCHA et al., 2005), fatores desmotivadores (BADDOO, 2001) e fatores de resistência (NASIR et al., 2008) relacionados à adoção de modelos de maturidade para melhoria de processos.

HABIB (2009) realizou um estudo empírico para identificar fatores críticos de sucesso, motivadores e obstáculos em iniciativas SPI em 4 países (Suécia, Paquistão, 
Dinamarca e Noruega). Os principais fatores críticos encontrados foram: comprometimento da alta gestão, envolvimento e experiência dos membros da organização, conscientização e implementação em SPI e alocação de recursos. Por sua vez, BAYONA et al. (2013) realizaram revisão sistemática para apontar fatores críticos de sucesso relacionados aos aspectos humanos. Alguns dos fatores identificados foram: presença de líderes na iniciativa de SPI, comprometimento da gerência, envolvimento da equipe de desenvolvimento e motivação.

VIRTANEN et al. (2013) identificaram que iniciativas de SPI costumam falhar, pois a alta direção costuma priorizar mais projetos em andamento do que o projeto de melhoria. Isso ocorre, pois a organização não aloca adequadamente recursos financeiros e tempo para o projeto de SPI. Em relação aos fatores desmotivadores, BADDOO (2001) identificou uma série de fatores, alguns dos exemplos são: imposição do processo, comunicação inadequada, falta de visibilidade em relação aos benefícios do projeto de melhoria e falta de recursos.

MONTONI (2010) concebeu um framework teórico constituído de conceitos e relacionamentos de influência, fundamentados em um conjunto de proposições (hipóteses), representando a visão e a perspectiva de implementadores de SPI. Foram identificados diversos fatores críticos de sucesso que influenciam iniciativas de SPI, por exemplo, "apoio, comprometimento e envolvimento", "aceitação às mudanças" e "motivação e satisfação dos membros da organização". Além dos fatores críticos de sucesso, MONTONI (2010) apresenta 41 tipos de achados de fatores de influência negativa, que foram identificados por meio da aplicação de procedimentos do método Grounded Theory (STRAUSS e CORBIN, 2008).

A aplicação de AO e GC em relação ao que deve ser feito nas atividades dos processos de software e sua melhoria são fatores críticos em Engenharia de Software (VIANA et al., 2012). A GC consiste em um processo de criação, captura, armazenamento e utilização do conhecimento de tal forma que ele possa ser transferido significativamente para outra pessoa (RAS et al., 2005). Segundo TIWANA (2002), o principal objetivo da GC no contexto organizacional é facilitar a aplicação oportuna de conhecimento fragmentado por meio da integração.

Segundo LAND et al. (2001), é fundamental que o conhecimento seja aprendido a nível organizacional, de tal forma que agregue sucesso às atividades de desenvolvimento de software realizadas. De acordo com SENGE (1991), Aprendizagem Organizacional (AO) pode ser definida como o ciclo contínuo de experiência e sua transformação em conhecimento acessível por toda organização e que seja relevante para suas finalidades básicas. Em outras palavras, AO é um processo adaptativo de mudança influenciado pela experiência no passado, e é centralizado no desenvolvimento ou modificação de rotinas e apoiado pela memória organizacional (MENOLLI, et al., 2016).

Neste contexto, VIANA (2015) desenvolveu o framework KL-SPI (Knowledge and Learning to Facilitate Software Process Improvement) que visa facilitar a $\mathrm{AO}$ e GC em organizações que implementam SPI. Este framework é composto por três componentes: (i) estratégia de diagnóstico do Estado da Prática de $\mathrm{AO}$ e GC nas organizações, (ii) catálogo de práticas de $\mathrm{AO}$ e $\mathrm{GC}$ e (iii) ferramentas para auxiliar a $\mathrm{AO}$ em SPI. VIANA (2015) definiu o catálogo de práticas de $\mathrm{AO}$ e $\mathrm{GC}$ a partir de investigações do estado da prática e de resultados de mapeamento sistemático literatura. 
Além da aplicação de GC, verifica-se que a utilização de práticas gerenciais para o tratamento de FCS de influência negativa é recomendada. ZANETTI et al. (2008) destacam que tratar iniciativas de melhoria como um projeto tradicional, ou seja, utilizando princípios de Gerência de Projetos, é importante para facilitar a percepção das melhorias obtidas a cada etapa do projeto e serve como incentivo para os membros da organização. Outra prática gerencial relevante consiste em homologar os processos com a alta direção. Este mecanismo é fundamental para o alinhamento e comprometimento da alta gerência e facilita a institucionalização dos processos (PORTO et al., 2008).

O presente trabalho concentra-se no tratamento dos 41 fatores críticos negativos identificados por MONTONI (2010), que podem ser encontrados, em sua íntegra, no Relatório Técnico ${ }^{1}$ correspondente a esse artigo. Decidiu-se escolher o trabalho de MONTONI (2010), pois nele foi feito um detalhamento e desdobramento sobre cada FCS, diferente dos demais trabalhos que apenas elencaram os fatores sem gerar categorias, associações e análises entre os FCS. Além disso, considerou-se o framework KL-SPI (VIANA, 2015), como referência para as práticas de GC e AO que visam tratar os fatores críticos negativos. Essas práticas foram definidas a partir da integração de investigações da prática com um mapeamento sistemático da literatura sobre AO e GC em Engenharia de Software e SPI. Desta forma, elas foram consideradas adequadas para o contexto desta pesquisa.

\section{Desenvolvimento da Pesquisa}

\subsection{Metodologia da Pesquisa}

A Figura 1 apresenta a abordagem metodológica deste trabalho, baseada no método Design Science Research (DSR) ou Pesquisa em Ciência do Design (WIERINGA, 2014). A abordagem DSR fundamenta e operacionaliza a condução da pesquisa quando o objetivo a ser alcançado é um artefato ou uma prescrição. Este método busca, a partir da compreensão do problema, construir e avaliar artefatos que permitam transformar situações, alterando suas condições para estados melhores ou desejáveis. Outra característica desta abordagem é ser orientada à solução de problemas específicos, não necessariamente buscando a solução ótima, mas a solução satisfatória para situação (DRESCH et al., 2015).

O artefato proposto é um catálogo constituído de práticas de Gerência de Conhecimento e Aprendizagem Organizacional, gerenciais e ferramentas que visa apoiar as organizações desenvolvedoras de software a confrontar com fatores críticos negativos durante uma iniciativa de SPI. No bloco "Estado da arte" estão os pilares deste estudo: o MR-MPS-SW (Modelo de Referência para Melhoria de Processos de Software) (SOFTEX, 2016) e o CMMI-DEV (Capability Maturity Model Integration for Development) (CMMI Product Team, 2010), análise qualitativa, o conjunto de fatores críticos de sucesso proposto por MONTONI (2010) e o framework KL-SPI concebido por VIANA (2015). O requisito principal do artefato é fornecer recomendações de práticas para as organizações mitigarem ou amenizarem o efeito de fatores que exerçam influência negativa em melhoria de processos de software. As

\footnotetext{
1 O Relatório Técnico encontra-se disponível no endereço: http://www.seer.unirio.br/index.php/monografiasppgi/article/view/6505
} 
atividades correspondentes a esta metodologia são apresentadas na Tabela 1. Essas atividades visam obter conhecimento em relação às práticas que podem ser utilizadas para tratar os fatores críticos negativos levantados por MONTONI (2010). Para isso, foram realizadas entrevistas com especialistas, investigação na literatura, entre outros estudos.

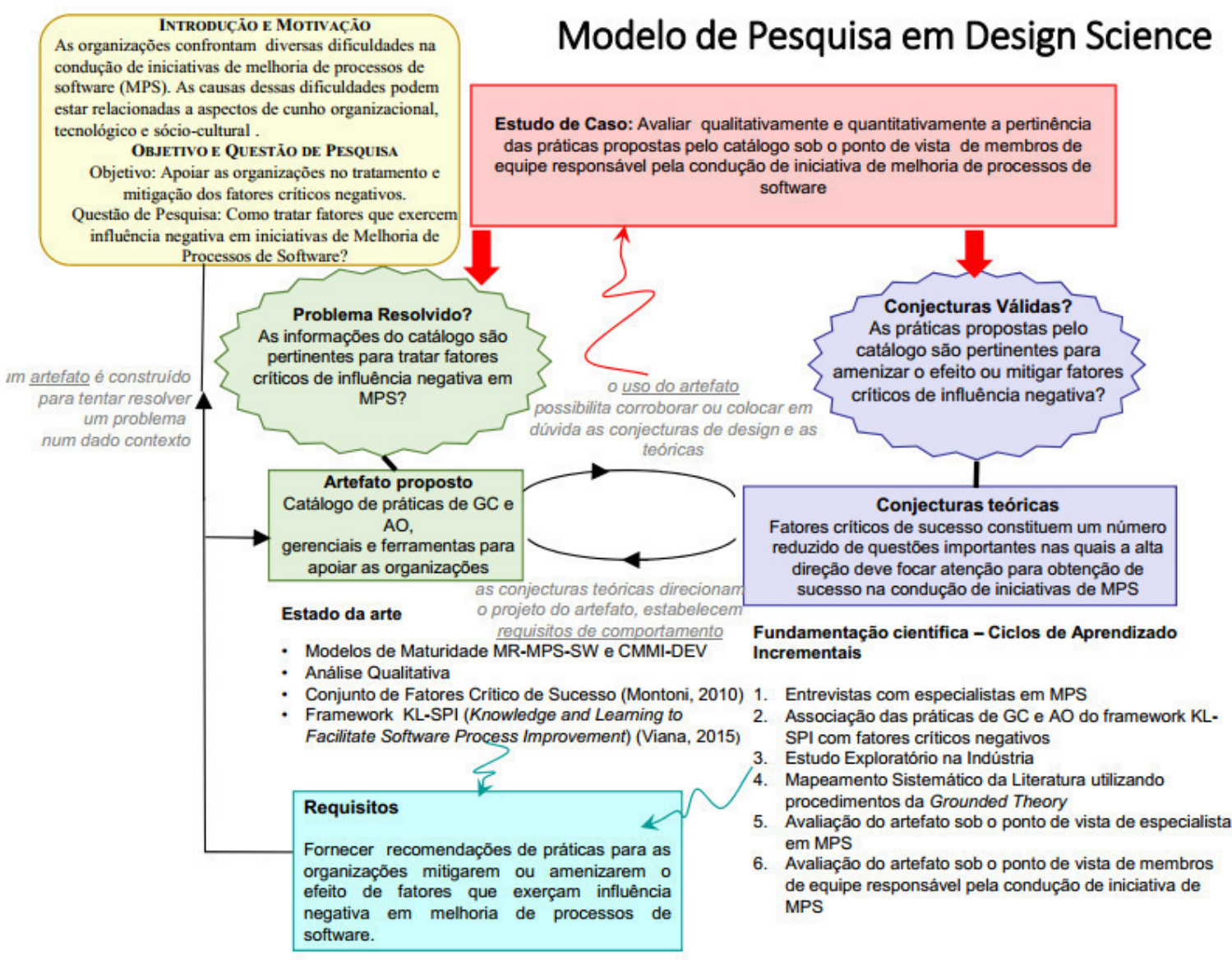

Figura 1.Modelo de pesquisa em Design Science utilizado neste trabalho

Tabela 1. Ciclos de aprendizado incrementais realizados durante a pesquisa

\begin{tabular}{|c|c|c|}
\hline Objetivo do ciclo & Principais ações & Principais achados \\
\hline $\begin{array}{ll}\text { Compreender } & \text { as } \\
\text { dificuldades } & \text { de } \\
\text { capacitação } & \text { de } \\
\text { implementadores } & \text { de } \\
\text { SPI } & \end{array}$ & $\begin{array}{l}\text { Realização de entrevistas e } \\
\text { aplicação de questionários } \\
\text { para consultores }\end{array}$ & $\begin{array}{l}\text { Identificados os processos que os consultores } \\
\text { possuem maior grau de dificuldade para avaliar e } \\
\text { implementar (exemplo: Aquisição- AQU e } \\
\text { Gerência de Portfólio de Projetos - GPP. Foram } \\
\text { verificados os tipos de conhecimento necessários } \\
\text { durante a implementação de uma iniciativa SPI }\end{array}$ \\
\hline $\begin{array}{l}\text { Verificar quais } \\
\text { práticas de GC e } \\
\text { AO poderiam ser } \\
\text { aplicadas para tratar } \\
\text { fatores críticos } \\
\text { negativos }\end{array}$ & $\begin{array}{l}\text { Associar as práticas de GC e } \\
\text { AO propostas pelo catálogo } \\
\text { de práticas do KL-SPI com os } \\
\text { fatores críticos negativos } \\
\text { apontados por MONTONI } \\
(2010)\end{array}$ & $\begin{array}{l}\text { Para o fator crítico "Alta rotatividade", foram } \\
\text { identificadas práticas como "Utilização de } \\
\text { ferramentas organizacionais/repositórios de } \\
\text { conhecimento", "Integração dos colaboradores", } \\
\text { "Interação com colaboradores experientes", } \\
\text { "Mentoring" e "Treinamento". Para os fatores } \\
\text { "Falta de motivação" e "Membros da equipe }\end{array}$ \\
\hline
\end{tabular}




\begin{tabular}{|c|c|c|}
\hline Objetivo do ciclo & Principais ações & Principais achados \\
\hline & & $\begin{array}{l}\text { insatisfeitos com a organização", foi sugerida a } \\
\text { prática "Premiação de projetos" }\end{array}$ \\
\hline $\begin{array}{llr}\text { Observar o } & \text { uso de } \\
\text { práticas de } & \text { GC } & \text { e } \\
\text { AO em um } & \text { contexto } \\
\text { real e, se possível, } \\
\text { com presença de } \\
\text { fatores r críticos } \\
\text { negativos }\end{array}$ & $\begin{array}{l}\text { Realização de estudo de caso } \\
\text { exploratório descritivo e } \\
\text { entrevista semiestruturada }\end{array}$ & $\begin{array}{l}\text { Identificou-se que as práticas "Utilização de } \\
\text { ferramentas organizacionais/repositórios de } \\
\text { conhecimento", "Aprender-fazendo", "Workshops" } \\
\text { e "Realização de acompanhamento/tutoria"auxiliam } \\
\text { no tratamento do fator crítico negativo "Alta } \\
\text { rotatividade", corroborando parcialmente com os } \\
\text { achados do ciclo anterior }\end{array}$ \\
\hline $\begin{array}{lr}\text { Investigar } & \text { na } \\
\text { literatura (relatos de } \\
\text { experiência e } & \text { artigos } \\
\text { técnicos) } & \text { mais } \\
\text { práticas } & \text { para } \\
\text { tratamento } & \text { de } \\
\text { fatores } & \text { críticos } \\
\text { negativos } & \\
\end{array}$ & 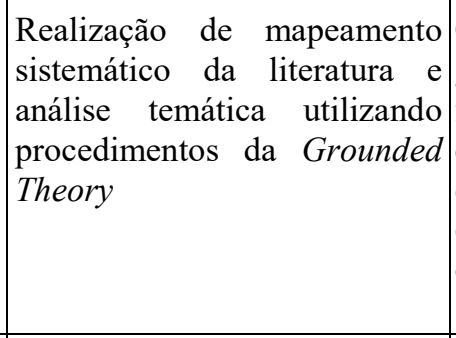 & $\begin{array}{l}\text { Obtidas ao todo } 186 \text { práticas, sendo } 135 \text { práticas } \\
\text { gerenciais, } 27 \text { práticas de GC e AO e } 24 \\
\text { ferramentas. Estas práticas foram divididas em } 8 \\
\text { categorias. Além disso, foram obtidas informações } \\
\text { estatísticas como quantidade de publicações por } \\
\text { evento, por organização, tipo de artigo e tipo de } \\
\text { organização }\end{array}$ \\
\hline$\left|\begin{array}{lrr}\text { Avaliar } & 0 \\
\text { mapeamento } & \text { entre } \\
\text { as práticas } & \text { sob } & 0 \\
\text { ponto de } & \text { vista } & \text { de } \\
\text { especialistas em } & \text { SPI }\end{array}\right|$ & 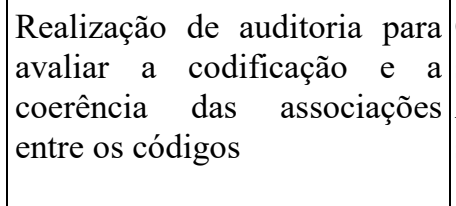 & $\begin{array}{l}\text { Os auditores recomendaram diversas práticas } \\
\text { sugestões para tratamentos dos fatores críticos. } \\
\text { Algumas práticas foram excluídas por não terem } \\
\text { sido consideradas adequadas }\end{array}$ \\
\hline
\end{tabular}

\subsection{Mapeamento Sistemático da Literatura}

Tendo em vista que nos três primeiros ciclos de aprendizado não se obteve um número expressivo de práticas para tratamento dos fatores críticos negativos, a motivação deste mapeamento sistemático da literatura consistiu em investigar na literatura a existência de mais práticas.

O planejamento deste estudo foi baseado nas diretrizes propostas por KITCHENHAM e CHARTERS (2007) e PETERSEN et al. (2015) para um mapeamento sistemático. O objetivo do mapeamento segue o paradigma Goal Question Metric (GQM) (BASILI e ROMBACH, 1988) e compreende: Analisar relatos de experiência e publicações científicas por meio de um mapeamento sistemático da literatura com o propósito de identificar práticas de Gerência de Conhecimento, de Aprendizagem Organizacional e gerenciais com relação a fatores críticos de influência negativa em iniciativas de melhoria de processos de software baseadas em um modelo de maturidade, como o MR-MPS-SW e o CMMI-DEV, do ponto de vista dos pesquisadores e profissionais envolvidos no contexto acadêmico e industrial

Para alcançar o objetivo descrito, foi definida a seguinte questão de pesquisa principal: "Como os fatores críticos de influência negativa são tratados em iniciativas de melhoria de processos de software?". Para responder essa questão foram estabelecidas quatro questões secundárias (QS):

- QS1. "Que práticas de Gerência de Conhecimento e Aprendizagem Organizacional foram utilizadas para tratar fatores críticos de influência negativa em iniciativas de SPI?" 
- QS2. "Que práticas gerenciais foram utilizadas para tratar fatores críticos de influência negativa em iniciativas de SPI?"

- QS3. "Que ferramentas foram utilizadas para tratar fatores críticos de influência negativa em iniciativas de SPI?"

- QS4. "Em que contexto as práticas e ferramentas identificadas foram utilizadas?"

É importante ressaltar que quando se trata de "fatores críticos de influência negativa" no âmbito deste trabalho, restringe-se aos 41 fatores críticos negativos identificados por MONTONI (2010). Logo, as questões de pesquisa apresentadas correspondem a este escopo de fatores críticos. Além disso, para responder a QS1, tomou-se como referência o catálogo de práticas de AO e GC de VIANA (2015).

Além disso, para responder às questões de pesquisa foi realizada uma análise temática utilizando alguns conceitos da Grounded Theory (STRAUSS e CORBIN, 2008). O método Grounded Theory (GT ou Teoria fundamentada a dados) consiste em um conjunto de procedimentos sistemáticos de coleta e análise dos dados para gerar, elaborar e validar teorias substantivas sobre fenômenos essencialmente sociais, ou processos sociais abrangentes (BANDEIRA-DE-MELLO e CUNHA, 2006). Este método compreende três fases: aberta, axial e seletiva. A codificação aberta envolve a quebra, a análise, a comparação, a conceituação e a categorização dos dados. Nesta fase, o pesquisador explora os dados, examinando minuciosamente aquilo que the parece relevante devido à leitura intensiva dos textos. Além disso, os incidentes ou eventos são agrupados em códigos através da comparação incidente-incidente. Após a identificação de categorias conceituais pela codificação aberta, a codificação axial examina as relações entre as categorias que formam as proposições da teoria substantiva (BANDEIRA-DE-MELLO e CUNHA, 2006).

Em relação ao escopo, foram selecionadas as conferências nacionais SBQS (Simpósio Brasileiro de Qualidade de Software) e WAMPS (Workshop Anual do MPS) e as internacionais PROFES (International Conference on Product-Focused Software Process Improvement) e EuroSPI (European Software Process Improvement). Esta escolha justifica-se, pois se tratam das conferências reconhecidas como as mais relevantes, na área de SPI, no contexto brasileiro (SBQS e WAMPS) e internacional (PROFES e EuroSPI). Em relação ao idioma, foram aceitos trabalhos escritos em português, inglês e espanhol, pois são os idiomas aceitos para publicação nas conferências selecionadas.

Foram identificados, ao todo, cerca de 1200 artigos, e, após aplicação dos filtros permaneceram 100 artigos, sendo 43 referentes ao SBQS, 34 ao WAMPS, 12 ao PROFES e 11 ao EuroSPI. Em relação à concentração de artigos selecionados ao longo do tempo, cabe destacar que houve um crescimento considerável no número de publicações entre 2005 e 2007. Isto pode ser explicado devido ao lançamento do modelo MR-MPS-SW, que ocorreu em 2005. Outro fator que contribui para este crescimento foi a realização dos eventos "Workshop de Instituições Organizadoras de Grupos de Empresas (IOGE's) MPS.BR" e "Workshop de avaliadores MPS.BR" entre os anos de 2006 e 2008.

Ao final da codificação aberta, foram obtidos ao todo 186 códigos referentes às práticas, sendo 135 práticas gerenciais, 27 práticas de $\mathrm{GC}$ e $\mathrm{AO}$ e 24 ferramentas. $\mathrm{O}$ passo seguinte foi realizar a codificação axial. Os 186 códigos gerados foram 
enquadrados em 8 categorias, conforme Tabela 2, onde também é apresentada a quantidade de práticas por categoria. Alguns códigos se enquadraram em mais de uma categoria. As descrições e detalhes sobre cada categoria são descritas no Relatório Técnico referente a esse artigo.

Tabela 2- Quantidade de Práticas por Categoria

\begin{tabular}{|c|c|c|}
\hline \# & Descrição da Categoria & $\begin{array}{l}\text { Quantidade } \\
\text { de práticas }\end{array}$ \\
\hline 01 & Estratégia de Implementação & 39 \\
\hline 02 & Melhoria Contínua do Processo & 18 \\
\hline 03 & Acompanhamento do Andamento das Atividades do Processo & 18 \\
\hline 04 & Colaboradores Possuírem Competências Adequadas para Execução das Atividades & 17 \\
\hline 05 & Definição, Validação e Padronização dos Processos e Procedimentos & 21 \\
\hline 06 & Conscientização, Incentivo e Divulgação do Processo & 38 \\
\hline 07 & Utilização de Ferramentas de Apoio & 28 \\
\hline 08 & Contexto Organizacional & 18 \\
\hline
\end{tabular}

Em relação à categoria "Acompanhamento do Andamento das Atividades do Processo" estão as práticas que visam acompanhar e monitorar o processo para que ele seja conduzido corretamente durante o andamento das atividades da iniciativa de SPI. Uma prática tipicamente utilizada é mentoring ou mentoria, que é uma técnica de acompanhamento assistido, conhecida também como treinamento "on the job". Esta técnica é normalmente realizada pela consultoria para acompanhar os membros da organização durante a utilização dos processos para dirimir dúvidas que possam surgir em relação ao seu uso (MONTEIRO et al., 2008). Muito similares a esta prática existem "Reuniões semanais para tirar dúvidas sobre o que está impedindo a execução do processo ou tem impacto na produtividade de uma determinada atividade" e "Grupo de Processos de Engenharia de Software (SEPG) atuar mais próximo da equipe de desenvolvimento" (FERREIRA et al., 2005; MENDES et al., 2011).

No contexto ágil, a adoção de práticas relacionadas com acompanhamento do andamento do processo também mostrou ser pertinente. SILVA et al. (2014) relatam que a participação do Grupo de Qualidade como um real integrante do time de desenvolvimento, durante as cerimônias Scrum, promoveu a aproximação do grupo de qualidade junto às equipes de desenvolvimento e facilitou a institucionalização do modelo de maturidade, combatendo então os fatores negativos "Falta de cultura de metodologia da organização" e "Grupo de qualidade isolado das equipes de desenvolvimento", respectivamente.

No que tange a categoria "Incentivo, Divulgação e Conscientização do Processo", FERREIRA et al. (2005) destacam que um dos mecanismos motivadores (fator negativo "Falta de motivação") é incentivar as equipes através de relatórios contendo indicadores de melhoria alcançados. PIRES et al. (2004) ressaltam que, para combater a resistência das equipes em relação à adoção dos novos processos (fator negativo "Cultura organizacional resistente a mudanças"), o artifício utilizado foi o envolvimento da alta gerência motivando as equipes por meio de palestras e oficinas. 


\subsection{Auditoria das Práticas sob o Ponto de Vista de Especialistas em SPI}

Finalizada a codificação, foram realizadas duas auditorias: a primeira consistiu em avaliar se os códigos gerados refletiam corretamente o conteúdo das citações e, a segunda, avaliar a coerência das associações entre os fatores negativos e as práticas indicadas para tratamentos destes. As auditorias foram baseadas em diretrizes propostas por BANDEIRA-DE-MELO e CUNHA (2010) e DUTRA (2015). Para evitar viés, foram selecionados auditores que não participaram do mapeamento sistemático da literatura, pois isso poderia influenciar nos resultados gerados.

$\mathrm{Na}$ primeira etapa da auditoria foram selecionados dois participantes, um com mestrado acadêmico concluído e outro em andamento na área de SPI com pesquisa voltada para análise qualitativa utilizando análise temática e GT. Além disso, os auditores receberam uma planilha que continha todos os códigos gerados na codificação aberta e espaço para indicar possíveis problemas identificados.

Ao final da primeira auditoria, foram identificados aproximadamente 15 erros de codificação, ou seja, um número pouco significativo em relação ao total de códigos. Entretanto, os auditores sugeriram diversas melhorias no texto dos códigos gerados, criação de notas explicativas (memos) para justificar alguns códigos e foram discutidas questões sobre os fatores críticos até que se chegasse a um entendimento comum.

Em relação à segunda parte da auditoria, o objetivo principal foi capturar a percepção de especialistas em SPI em relação às associações entre as práticas e os fatores negativos que foram realizadas durante a análise temática utilizando procedimentos de GT. Nesta etapa foram selecionados também dois participantes: um pós-doutorando com doutorado ligado a SPI, implementadora e avaliadora líder intermediária credenciada do modelo de referência MR-MPS-SW e MR-MPS-SV e membro de Instituição Implementadora e Avaliadora de melhoria de processos. O segundo possui doutorado em Engenharia de Software com pesquisa voltada para Engenharia de Software e Gerência do Conhecimento, docente e pesquisador e que já ministrou disciplina de Gestão da Qualidade de Software. Além disso, possui conhecimento comprovado no modelo MR-MPS-SW, além de ter participado de implementações MR-MPS-SW.

Semelhantemente à auditoria 1 , nesta etapa os auditores receberam dois arquivos: um relatório, exportado da ferramenta Atlas. $\mathrm{Ti}^{2}$, que continha os fatores críticos negativos e suas práticas correspondentes, e (ii) uma planilha contendo os fatores críticos negativos e uma pergunta para avaliar a coerência das associações entre os códigos.

Para cada um dos 41 fatores negativos, o auditor deveria responder o seguinte questionamento "Existem elementos que levam a acreditar que a adoção das práticas (de GC/AO, gerencial ou ferramenta) pode ser utilizada no tratamento do fator negativo?". Os auditores deveriam responder "Sim" ou "Não", justificando o erro, caso ocorresse, e dispunham de espaço para sugerir correções.

Ao final desta auditoria, foram obtidas diversas sugestões para as recomendações indicadas para tratamentos dos fatores críticos. Os auditores apontaram práticas que deveriam ser acrescentadas ou excluídas. Por exemplo, para o fator

\footnotetext{
${ }^{2}$ Atlas.Ti é uma de análise qualitativa de dados (Site: atlasti.com)
} 
negativo "Equipes diferentes de desenvolvimento não querem executar o processo da mesma forma", um dos auditores sugeriu incluir as práticas "Permitir adaptações no processo padrão para cada projeto, respeitando as necessidades de cada projeto e as adaptações permitidas pela empresa" e "Grupo de Garantia da Qualidade avaliar os produtos gerados e a aderência ao processo".

Em relação aos fatores negativos "Imposição do processo às pessoas que devem executá-lo" e "Mudança de procedimentos de execução dos processos implementados" foi sugerida a prática "Apresentar periodicamente para todos o andamento do projeto, quais são os objetivos pretendidos e quais são os passos para o alcance das metas". E para "Alta rotatividade" foi indicada a prática "Existência de um processo padrão formal para que todos adotem os mesmos procedimentos e estejam cientes de suas responsabilidades". As sugestões foram examinadas e o artefato sofreu as adaptações necessárias.

\section{Catálogo de Práticas para Tratamento de Fatores Críticos Negativos}

\subsection{Estrutura do Catálogo}

Finalizados os ciclos de aprendizado incrementais apresentados nas seções anteriores, o catálogo de práticas de $\mathrm{GC}$ e $\mathrm{AO}$ e ferramentas foi confeccionado de tal forma que foram unificadas todas as práticas identificadas. Buscou-se gerar um instrumento com a finalidade de apoiar as organizações, mais especificamente o grupo de processos ou grupo responsável pela implementação, a tratar fatores negativos já existentes ou mitigar fatores que possam vir a surgir no futuro.

Este catálogo também é útil para consultores de Instituições Implementadoras (IIs) do modelo MR-MPS-SW, pois em determinados contextos, a equipe de processos da organização pode não possuir o nível de experiência adequado para tomada de decisão em situações negativas no decorrer do projeto de melhoria. Então, as recomendações dadas pelo catálogo poderão nortear as ações dos consultores.

O Catálogo é formado por um total de 35 fichas, cada uma corresponde a um fator crítico negativo. A Figura 2 apresenta uma das fichas, que é referente ao fator crítico negativo "Equipes diferentes de desenvolvimento não quererem executar o processo da mesma forma".

$\mathrm{Na}$ primeira linha da ficha é apresentado o nome do fator crítico negativo, logo abaixo, a categoria a que este fator pertence. Em seguida, são exibidos os 3 blocos principais que formam o catálogo: "Práticas de GC e AO", "Práticas gerenciais" e "Ferramentas" que são indicadas para tratamento do fator. Em cada um dos blocos é diferenciado se a prática teve ou não evidência de uso. As práticas que foram classificadas em "Práticas com evidência de uso" são aquelas onde o autor da publicação explicitou que houve uso de tal prática. O Relatório Técnico referente a esse artigo apresenta a relação completa de todas as práticas com as respectivas referências e evidências de uso. Abaixo dos blocos, são indicados os contextos onde as práticas foram aplicadas (no exemplo em questão, CMMI-DEV nível 2 e 3 e MR-MPS-SW nível G e F). Por último, são apresentadas as referências das práticas, ou seja, as fontes que as respaldam. 


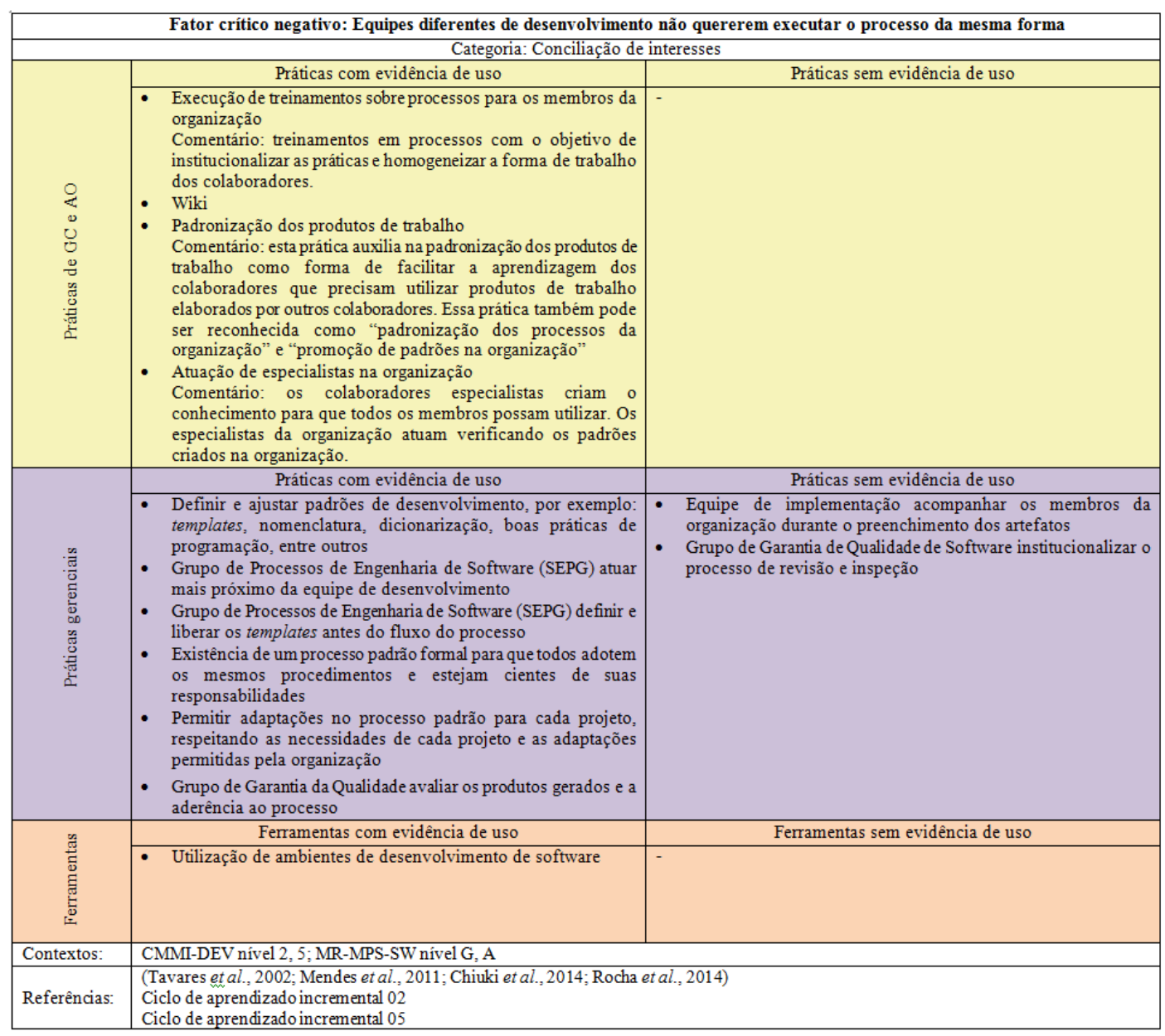

Figura 2. Exemplo de ficha do Catálogo

No exemplo em questão, as práticas gerencias "Equipe de implementação acompanhar os membros da organização durante o preenchimento dos artefatos" e "Grupo de Garantia de Qualidade de Software institucionalizar o processo de revisão e inspeção" são oriundas do $4^{\circ}$ ciclo, então são indicadas as referências da literatura “(Tavares et al., 2002; Mendes et al., 2011; Chiuki et al., 2014; Rocha et al., 2014)". As práticas de GC e AO "Wiki", "Padronização dos produtos de trabalho" e "Atuação de especialista" foram geradas a partir do Ciclo de aprendizado 02 e "Permitir adaptações no processo padrão para cada projeto, respeitando as necessidades de cada projeto e as adaptações permitidas pela organização" foi uma sugestão dada por um dos especialistas no Ciclo de aprendizado 05.

\subsection{Discussão sobre o Catálogo}

A Figura 3 apresenta um mapa mental do Catálogo de forma sintetizada. Do lado esquerdo encontram-se alguns dos fatores críticos negativos, e à direita parte das práticas de $\mathrm{GC}$ e $\mathrm{AO}$, práticas gerenciais e ferramentas para tratamento destes fatores. Percebe-se que para alguns fatores, como "Alta rotatividade de pessoal", não foram identificadas ferramentas para tratar o fator. 


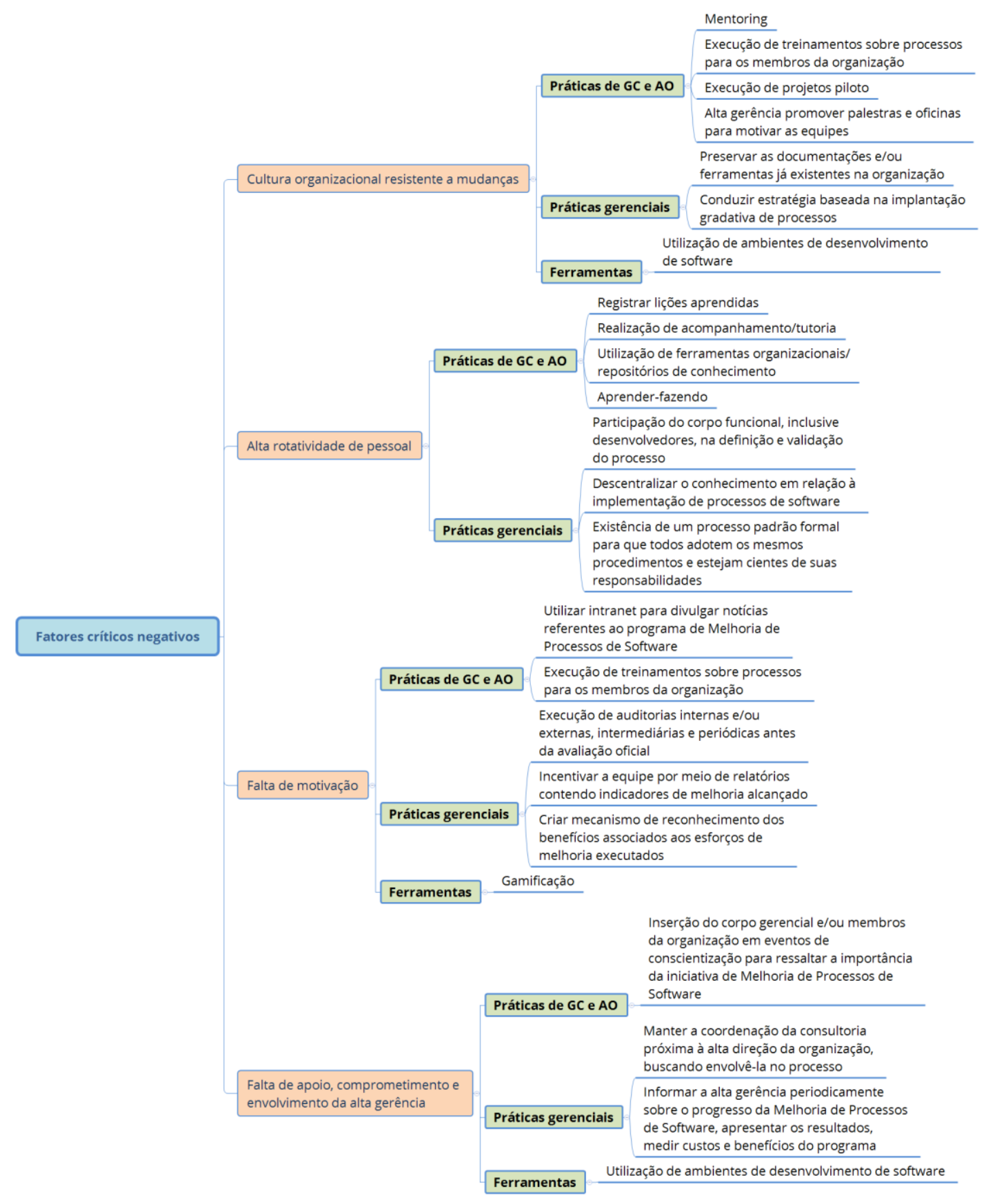

Figura 3. Mapa mental sintetizado do Catálogo

Um ponto importante a se destacar em relação ao Catálogo é que algumas práticas foram indicadas para mais de um fator negativo. Uma prática gerencial que se enquadra nessa situação é "Execução de auditorias internas e/ou externas, intermediárias e periódicas antes da avaliação oficial". Em algumas publicações, autores relatam que cada vez que essa prática era aplicada percebiam-se novas oportunidades de melhoria no processo e os ajustes eram realizados no ciclo posterior, contribuindo então para a adequação do processo (MARCZAK et al., 2003). Já em ROCHA et al. (2005), notou-se elevação nos níveis de motivação ao realizar auditorias internas, pois foi gerado um clima de competição saudável no qual todos fiscalizavam todos. Por outro lado, em um contexto de desenvolvimento utilizando práticas ágeis identificou-se que 
auditorias constantes provocaram maior atenção dos colaboradores em seguir os processos (SILVA et al., 2014).

DRESCH et al. (2015) ressaltam que um dos fatores fundamentais para o sucesso de uma pesquisa conduzida com DSR é a relevância da pesquisa para as organizações. Dessa forma, após construção do catálogo, verificou-se a necessidade de avaliá-lo sob o ponto de vista de organizações que participaram de uma iniciativa SPI. Para isso, foram realizados dois estudos de caso em diferentes organizações para analisar o catálogo de forma quantitativa e qualitativa. $O$ participante do estudo teve alto grau de envolvimento com os projetos de melhoria, tendo exercido o papel de coordenador do grupo de processos e líder de qualidade. Após execução das entrevistas e preenchimento de questionários, foi obtido um grau de $84 \%$ das práticas em relação às iniciativas de SPI em questão.

Dessa forma, observa-se que o catálogo poderá auxiliar as organizações, mais especificamente o grupo de processos ou grupo responsável pela implementação, a tratar fatores negativos já existentes ou mitigar fatores que possam vir a surgir no futuro. Este catálogo também é útil para consultores de IIs, pois em determinados contextos, a equipe de processos da organização pode não possuir o nível de experiência adequado para tomada de decisão em situações negativas no decorrer do projeto de melhoria. Então, as recomendações dadas pelo catálogo poderão nortear as ações dos consultores. O catálogo é útil também para a alta direção da organização, pois contém diversas práticas que direcionam o alinhamento do projeto de melhoria com os objetivos estratégicos da organização. Além disso, como as práticas potencializam as chances de sucesso da iniciativa de SPI, então como consequência, aumentam-se as chances de a organização obter vários benefícios, como aumento do lucro, ROI (retorno sobre o investimento), aumento da satisfação dos clientes, entre outros.

As principais limitações deste trabalho são:

- Alguns fatores críticos negativos, como "Disputas internas (políticas) dentro da organização a ser avaliada" e "Falta de abertura do líder de qualidade para ouvir outras opiniões" não foram contemplados devido à sua natureza. Não foram sugeridas práticas para estes fatores, pois envolvem variáveis difíceis de serem controladas, como fatores políticos da organização ou fatores psicológicos de membros da organização;

- Não considerar, no escopo do mapeamento sistemático da literatura, artigos publicados de outros eventos, como ESELAW (Experimental Software Engineering Latin America Workshop), AGILE (Agile Process in Software Engineering and Extreme Programming), periódicos e journals como Software Quality Journal, Journal of Systems and Software e Software Practice and Experience.

\section{Considerações Finais e Trabalhos Futuros}

Tendo em vista que as organizações que desenvolvem software e implementam um modelo de maturidade são suscetíveis a uma série de fatores e riscos que podem prejudicar ou comprometer a iniciativa de melhoria, este trabalho se propôs a identificar práticas que podem ser utilizadas para tratar estes problemas. Para isso, optou-se pela metodologia DSR, onde foram realizados 6 ciclos de aprendizado incrementais, que 
consistiram em cinco avaliações intermediárias e, por último, uma avaliação final para obter a percepção de um membro responsável pela condução de uma iniciativa de SPI.

A principal contribuição deste trabalho foi a geração de um catálogo com 135 práticas gerenciais, 27 práticas de $\mathrm{GC}$ e $\mathrm{AO}$ e 24 ferramentas para tratamento de fatores críticos de influência negativa. Este instrumento pode ser utilizado por organizações desenvolvedoras de software como insumo para elaboração de um plano de ação (ou plano de risco) durante a iniciativa de SPI. É recomendado que a organização tenha um plano de gestão de riscos antes mesmo do início do projeto de melhoria (DUTRA, 2015).

Alguns possíveis trabalhos futuros incluem: (i) realizar experimentos para verificar a efetividade de práticas que foram identificadas no catálogo, (ii) realizar entrevistas com diversos implementadores e consultores credenciados pelo modelo de referência MPS.BR para capturar opinião em relação aos resultados gerados no catálogo, (iii) aplicar o estudo de caso em outras organizações que estejam planejando ou executando uma iniciativa de SPI, e capturar a opinião não apenas de membros da organização, mas também de consultores que participam (ou participaram) do projeto de melhoria e (iv) incluir outras fontes no escopo do mapeamento sistemático da literatura.

\section{Agradecimentos}

Os autores agradecem à FAPERJ (projetos E-26/210.643/2016, E-211.174/2016) e à UNIRIO (Edital PQ-UNIRIO no. 01/2016 e 01/2017) pelo apoio financeiro. O Segundo autor agradece à UFMA através do Projeto PVCET201-2016.

\section{Referências}

BANDEIRA-DE-MELLO, R., CUNHA, C., 2006, "Grounded Theory". In: GODOI, C.K., BANDEIRA-DEMELlO, R., SILVA, A.B.D. (eds), Pesquisa Qualitativa em Estudos Organizacionais: Paradigmas, Estratégias e Métodos, São Paulo, Saraiva.

BANDEIRA-DE-MELLO, R., CUNHA, C., 2010, “Grounded Theory”. In: GODOI, C. K., BANDEIRA-DEMELLO, R., SILVA, A.B. (eds), Pesquisa Qualitativa em Estudos Organizacionais: Paradigmas, Estratégias e Métodos, Chapter 8, 2a ed, São Paulo, Saraiva.

BASILI, V., ROMBACH, H., 1988, "The Tame Project: Towards Improvement-Oriented Software Environments", IEEE Transactions on Software Engineering, v.14, n. 6, pp. 758-773

BAYONA, S., CALVO-MANZANO, J. A., FELIU, T. S. (2012) Critical Success Factors in Software Process Improvement: A Systematic Review. 12th International Conference, SPICE 2012, Palma, Spain, May 29-31, 2012.

BAYONA, S., CALVO-MANZANO, J. A., FELIU, T. S. (2013). Review of Critical Success Factors Related to People in Software Process Improvement. 20th European Conference, EuroSPI 2013, Dundalk, Ireland, June 25-27, 2013. Proceedings.

CMMI PRODUCT TEAM, 2010, CMMI® for Development (CMMI-DEV), V1.3, Software Engineering Institute. Disponível em: http://cmmiinstitute.com/resources/cmmi-development-version-13. Acessado em 18 de dezembro de 2015.

DRESCH, ALINE; LACERDA, D. P. ; ANTUNES JUNIOR, J. A. V. . Design Science Research: Método de Pesquisa para Avanço da Ciência e Tecnologia. 1. ed. Porto Alegre: Bookman, 2015. v. 1. 181p

DUTRA, E. Riscos em iniciativas de melhoria de processos de software baseadas no MR-MPS-SW e no CMMIDEV: uma investigação no contexto brasileiro. 2015. XIV Simpósio Brasileiro de Qualidade de Software, SBQS 2015, Manaus, AM .

FERREIRA, A. I. F., CERQUEIRA, R., ROCHA, A. R., et al. (2005) "Implantação de Processo de Software na BL Informática - Um Caso de Sucesso". IV Simpósio Brasileiro de Qualidade de Software, SBQS 2005, Porto Alegre, RS.

FUGgetTA, A. (2000) Software Process: A Roadmap, In: Proceedings of The Future of Software Engineering, ICSE2000, Limerick, Ireland.

IANDOLI, L., G. ZOLLO, Organization cognition and learning: building systems for the leaning organization, Inf. Sci. Publ. (2008). 
KITCHENHAM, B. E CHARTERS, S., 2007, "Guidelines for performing Systematic Literature Reviews in Software Engineering”, Technical Report EBSE 2007-001, Keele University and Durham University Joint Report, 2007.

MARCZAK, SABRINA; SÁ, L. ; CECCATO, I. ; AUDY, J. L. N. ; ANTUNES, D. . Uma proposta de organização e funcionamento da função de Garantia da Qualidade de Software em um contexto de implantação do SWCMM. In: II Simpósio Brasileiro de Qualidade de Software, 2003, Fortaleza, CE. Anais do Simpósio Brasileiro de Qualidade de Software. Fortaleza, CE: UNIFOR, 2003. p. 1-12.

MENDES, F. F., OLIVEIRA, J. L., FERNANDES, P. G., SOUZA, A. S. (2007) "Análise de Riscos na Implantação de Melhorias de Processos de Software". In: ProQualiti - Qualidade na Produção de Software, v. 3, nro. 3, pp. 25-32, Nov/2007.

MENDES, F. F., ALMEIDA, J. N., ARRUDA JUNIOR, E. Experiência de Implantação de Melhoria de Processos de Software em um Laboratório de Pesquisa. In: Workshop Anual do MPS, 2011, Campinas. VII Workshop Anual do MPS - WAMPS 2011, 2011.

MENOLLI, ANDRÉ LUÍS ANDRADE; PINTO, H. SOFIA ; REINEHR, SHEILA ; MALUCELLI, ANDREIA . SECOL: a semantic environment based on social media to support organisational learning. BEHAVIOUR \& INFORMATION TECHNOLOGY, v. 36, p. 1-26, 2016.

MONTEIRO, R. W., Cabral, R., Alho, F., et al. (2008) "O Esforço Requerido para Institucionalização de Processos de Software na Prodepa". In: ProQualiti - Qualidade na Produção de Software, v. 4, nro. 2, pp. 65-72, Out/2008.

MONTONI, M., 2010, Uma investigação sobre os fatores críticos de sucesso em iniciativas de melhoria de processos de software, Tese de D.Sc., Universidade Federal do Rio de Janeiro - UFRJ, Rio de Janeiro, RJ, Brasil.

MONTONI, M. A., ROCHA, A. R., 2011, "Uma Investigação sobre os Fatores Críticos de Sucesso em Iniciativas de Melhoria de Processos de Software". In: Proceedings of X Simpósio Brasileiro de Qualidade de Software, v. 1, pp. 1-15, Curitiba, PR.

NASIR, M.H.N.M., AHMAD, R., HASSAN, N.H., 2008, "Resistance factors in the implementation of software process improvement project", Telematics and Informatics v. 3, pp. IEEE, Kuala Lumpur, Malaysia

PARENTE, T. M. G., ALBUQUERQUE, A. B. (2008) "Domínio Informática: a qualidade como foco do seu Plano Estratégico". In: ProQualiti - Qualidade na Produção de Software, v. 4, nro. 2, pp. 47-52, Out/2008.

PETERSEN, K., VAKKALANKA, S., KUZNIARZ, L., 2015, "Guidelines for conducting systematic mapping studies in software engineering: An update". Information and Software Technology, Num. 64, pp. 1-18.

PIRES, C. G., Marinho, F., Telles, G., Belchior, A. (2004) "A Experiência de Melhoria do Processo do Instituto Atlântico Baseado no SW-CMM nível 2". III Simpósio Brasileiro de Qualidade de Software, SBQS 2004, Brasília, Distrito Federal.

PORTO, Josiane Brietzke; PEREIRA, A. C. ; POHREN, Juliana . Proposta de Melhoria de Processos da SWB Soluções Integradas usando o MR-MPS e a abordagem PRO2PI. ProQuality (UFLA), v. 4, p. 57-64, 2008.

RAS, E.; M. MEMMEL, S. WEIBELZAHL, Integration of E-Learning and Knowledge Management - Barriers, Solutions and Future Issues, in: Proceedings of third Conference Professional Knowledge Management Experiences and Visions, Berlin, 2005.

ROCHA, Ana Regina ; MONTONI, Mariano ; SANTOS, G. ; MAFRA, Sômulo ; FIGUEIREDO, Sávio ; ALBUQUERQUE, Adriano Bessa ; MIAN, Paula . Estação TABA: Uma Infra-estrutura para Implantação do Modelo de Referência para Melhoria de Processo de Software. In: IV Simpósio Brasileiro de Qualidade de Software, 2005, Porto Alegre - RS. Anais IV Simpósio Brasileiro de Qualidade de Software, 2005. p. 49-60.

SENGE, P.M., 1991, "The fifth discipline, the art and practice of the learning organization", Performance + Instruction, v. 30, n. 5, pp. 37-37.

SILVA, N. V., Antiquera, P. R. S.,. Burity, E. R. Encontrando o Equilíbrio entre a Metodologia Scrum na Fábrica JAVA e o modelo MPS.br- SW - nível F. In: Workshop Anual do MPS.BR, 2014, Campinas. X Workshop Anual do MPS.BR (WAMPS 2014). Campinas - SP: SOFTEX - Associação para Promoção da Excelência do Software Brasileiro, 2014.

SOFTEX, 2016, MPS.BR: Guia Geral MPS de Software, Disponível em: http://www.softex.br/mpsbr/guias/. Acessado em 14 de janeiro de 2016.

STRAUSS, A., CORBIN, J., 2008, Pesquisa Qualitativa - Técnicas e Procedimentos para o desenvolvimento de teoria fundamentada. 2a. Ed., Porto Alegre: Artmed e Bookman.

TIWANA, A. Knowledge Management Toolkit, Person Education, 2002.

VIANA, D., Conte, T., Vilela, D., et al., 2012, "The influence of human aspects on software process improvement: Qualitative research findings and comparison to previous studies". In: EASE 2012, on, pp. 121-125, 14-15 May 2012

VIANA, D. "FACILITANDO A APRENDIZAGEM ORGANIZACIONAL EM MELHORIA DE PROCESSO DE SOFTWARE". Tese de Doutorado - UNIVERSIDADE FEDERAL DO AMAZONAS - UFAM. Manaus, Amazonas, Março de 2015.

VIRTANEN, P., PEKKOLA, S. and PÄIVÄRINTA, T. "Why SPI initiative failed: contextual factors and changing software development environment."System Sciences (HICSS), 2013 46th Hawaii International Conference On. IEEE, 2013

WIERINGA, R., 2014, "Design Science Methodology for Information Systems and Software Engineering”, Springer Heidelberg, ISBN 978-3-662-43839-8, 332pp. 